\title{
The Relationship between Estimated Glomerular Filtration Rate and Diabetic Retinopathy
}

\author{
Jingyang Wu, ${ }^{1}$ Jin Geng, ${ }^{1}$ Limin Liu, ${ }^{1}$ Weiping Teng, ${ }^{2}$ Lei Liu, ${ }^{1,3}$ and Lei Chen ${ }^{1,2}$ \\ ${ }^{1}$ Department of Ophthalmology, The First Hospital of China Medical University, Shenyang 110001, China \\ ${ }^{2}$ Key Laboratory of Endocrine Diseases of Liaoning Province, The First Hospital of China Medical University, Shenyang 110001, China \\ ${ }^{3}$ Department of Epidemiology, School of Public Health, China Medical University, Shenyang 110001, China
}

Correspondence should be addressed to Lei Liu; leiliuyang@163.com and Lei Chen; leichen0501@163.com

Received 30 December 2014; Revised 5 March 2015; Accepted 8 March 2015

Academic Editor: Tamer A. Macky

Copyright (C) 2015 Jingyang Wu et al. This is an open access article distributed under the Creative Commons Attribution License, which permits unrestricted use, distribution, and reproduction in any medium, provided the original work is properly cited.

\begin{abstract}
Diabetic retinopathy (DR) is the leading cause of visual impairment and blindness in working-aged people. Several studies have suggested that glomerular filtration rate (GFR) was correlated with DR. This is a hospital-based study and the aim of it was to examine the relationship between the GFR and DR in patients with type 2 diabetes mellitus (T2DM). We used CKD-EPI equation to estimate GFR and SPSS 19.0 and EmpowerStats software to assess their relationship. Among the 1613 participants (aged 54.75 \pm 12.19 years), 550 (34.1\%) patients suffered from DR. The multivariate analysis revealed that the risk factors for DR include age $(P<0.001, \mathrm{OR}=0.940)$, duration of diabetes $(P<0.001, \mathrm{OR}=1.163)$, hemoglobin Alc $(P=0.007, \mathrm{OR}=1.224)$, systolic blood pressure $(P<0.001, \mathrm{OR}=1.032)$, diastolic blood pressure $(P=0.007, \mathrm{OR}=0.953)$, high density lipoprotein cholesterol $(P=0.024$, $\mathrm{OR}=3.884)$, and eGFR $(P=0.010, \mathrm{OR}=0.973)$. Through stratified analysis and saturation effect analysis, our data suggests that eGFR of $99.4 \mathrm{~mL} / \mathrm{min}$ or lower might imply the early stage of DR in diabetic patients. Thus, the evaluation of eGFR has clinical significance for the early diagnosis of DR.
\end{abstract}

\section{Introduction}

The prevalence of type 2 diabetes mellitus (T2DM) has significantly increased worldwide in the past 25 years $[1,2]$. DR is one of the most common microvascular complications of diabetes mellitus (DM) and is the leading cause of visual impairment and blindness in working-aged people. In recent years, the prevalence of DR is rapidly increasing $[3,4]$, as the number of people with T2DM increased. The results of a cross-sectional study in a multiethnic Asian population showed that the overall age-standardized prevalence of DR was $25.4 \%(20 \%, 24.8 \%$, and $28.9 \%$ in Chinese, Malays, and Indians, resp., $P=0.290$ ) [5]. More than $50 \%$ of T2DM patients likely suffer from DR within twenty years after diagnosis [6]. Because the symptoms of DR are not apparent in early stages of this disease, patients often miss the best opportunity for treatment when diagnosed, leading to a high rate of blindness. According to the World Health Organization (WHO), DR accounts for $4.8 \%$ of the total cases of blindness (thirty-seven million worldwide) in 2006 [7]. Therefore, it is important to investigate the risk factors that promote or predict DR.

Diabetic nephropathy (DN), also known as diabetic kidney disease or diabetic glomerulosclerosis, is another major complication of DM and the leading cause of end-stage renal disease (ESRD). GFR and microalbuminuria are clinically significant markers for the evaluation of renal function. Previous studies have shown that microalbuminuria not only is an important clinical marker for DN, but also is closely associated with the progression of DR [8]. However, suffering from DR and the appearance of microalbuminuria do not occur at the same time. GFR describes the flow rate of filtered fluid through the kidney and can be estimated using formulas, thereafter referred to as estimated GFR (eGFR). Unlike microalbuminuria, GFR increases during the early stages of DM due to high blood sugar and decreases during the later stages of DM, reflecting a decline in renal function. That is to say, changes in GFR appear earlier than microalbuminuria in diabetic patients. Past studies have reported that GFR is but one variable of many that affects the likelihood of developing 
DR and the other complications of $\operatorname{DM}[9,10]$. In addition, due to the limited medical condition, routine funduscopic examination or microalbuminuria cannot be performed in primary hospital in rural China, especially poverty-stricken areas. So we wondered if eGFR could be used for the early detection of DR, in order to screen it in the general population. If possible, eGFR could be used for screening in the general population.

Hence, our researchers are embarking on a series of studies to investigate the relationship between GFR and DR, and this is the baseline study. The aim of this study was to evaluate the prevalence of DR in hospital-based T2DM patients and investigate the correlation between GFR and DR, so that GFR could be used for DR screening, especially in primary hospital of poor areas.

\section{Materials and Methods}

2.1. Study Participants. We conducted a hospital-based casecontrol study. DM patients were admitted to the Department of Endocrinology and Ophthalmology of the First Affiliated Hospital in China Medical University from September 2010 to March 2012. A total of 1613 patients, 844 (52.3\%) males and 769 (47.7\%) females, with T2DM were enrolled in this study after rigorous diagnosis and exclusion criteria. The project was performed in accordance with the principles of the Declaration of Helsinki and approved through the Ethics Committee of China Medical University.

2.2. Diagnosis and Exclusion Criteria. Diabetes was diagnosed according to the 2006 World Health Organization (WHO) criteria [11]. The DR severity was classified according to the International Clinical Diabetic Retinopathy Disease Severity Scale [12]. Patients were excluded if they had type 1 diabetes mellitus, acute metabolic disorders (such as diabetic ketoacidosis and a hyperglycemic hyperosmolar state), opaque refractive media of one or both eyes (affecting fundus observation), or other eye diseases or serious illnesses (such as cancer). Because DN is often complicated with DR [13], these patients were not excluded to avoid selection bias.

2.3. Research Design and Evaluation. The clinical data were extracted from the medical records of 1613 T2DM patients. Information, including the age, gender, duration of diabetes, family history of diabetes, and history of hypertension, was collected for each patient. The blood pressure was measured during the medical examination and recorded as the means of two measurements after the patients rested for 5 minutes. Fasting blood was drawn from the cubital vein and used for biochemical assays, including fasting blood glucose (FBG), glycosylated hemoglobin (HbAlc), triglycerides (TG), total cholesterol (TC), high density lipoprotein cholesterol (HDLC), low-density lipoprotein cholesterol (LDL-C), serum creatinine (Scr), and blood urea nitrogen (BUN). For the 2hour postprandial glucose (2hPG) measurement, blood was drawn at 2 hours after ingestion of $75 \mathrm{~g}$ glucose powder or bread (equivalent amount of carbohydrates). All samples were measured using the ARCHITECT c8000 biochemical analyzer (Toshiba, Tokyo, Japan). The eGFR was calculated using the CKD-EPI equation [14]. The unit of SCR in the CKD-EPI formula should be "mg/dL," while the levels of SCR through colorimetric method were in " $\mu \mathrm{mol} / \mathrm{L}$." Therefore, we converted SCR according to the formula " $1 \mathrm{mg} / \mathrm{dL}=$ $17.1 \mu \mathrm{mol} / \mathrm{L}$ " and put it into the formula to obtain eGFR.

Direct ophthalmoscopy and fundus photography were conducted after the participants were administered mydriatic eye drops to dilate the pupils. The center of the macula and optic disc of both eyes was photographed using a 45-degree digital camera (CR-DGI, Canon, Japan). Two trained ophthalmologists performed the diagnosis of DR. If there were different opinions, they would discuss to make decision or consult the superior. Based on the results of the assessment, the subjects were divided into two groups: a DR group and a non-DR (DM without DR) group. There were 550 patients in DR group (male 269, female 281) and 1063 patients in NDR group (male 575, female 488 ).

2.4. Statistical Analysis. All statistical analyses were performed using the Statistical Package from the Social Sciences (SPSS) version 19.0, EmpowerStats (http://www.empowerstats.com/), and MedCalc software programs. The basic description and logistic regression analyses were performed using SPSS 19.0 software. The stratified analysis, the interaction test, covariate screening, and curve fitting were performed using EmpowerStats statistical software. MedCalc was used to draw the receiver operating characteristic curve (ROC curve), a graphical plot illustrating the performance of a binary classifier system with varying discrimination thresholds. The area under the ROC curve (AUC) ranged from 0.5 to 1 . The numerical variables of normal distribution were expressed as the means \pm standard deviation (means \pm SD) and percentage (\%). The independent samples $t$-test was used to analyze the continuous variables, whereas the odds ratio (OR) and chi-square $\left(\chi^{2}\right)$ test was used to analyze the categorical variables. $P<0.05$ was considered as statistically significant.

\section{Results}

Based on the exclusion criteria, 1613 T2DM patients aged $54.75 \pm 12.19$ years were selected into this study. Among these subjects, 550 (34.1\%) patients were diagnosed with DR. No differences in TG, DBP, and BUN were detected between the DR and NDR groups. In contrast, statistically significant differences were detected for age $(P=0.003)$, family history of DM $(P=0.036)$, duration of DM $(P<0.001)$, FBG and 2hPG $(P<0.001)$, HbAlc $(P=0.003)$, TC $(P<0.001)$, HDL-C $(P=0.013)$, LDL-C $(P=0.002)$, SBP $(P<0.001)$, DBP $(P=0.033)$, eGFR $(P<0.001)$, and SCR $(P<0.001)$ (Table 1).

To determine the risk factors for $\mathrm{DR}$, a logistic regression model was performed. DR was used as the dependent variable. Different risk factors, identified in the univariable analysis, were used as independent variables (Table 2). There were significant associations between DR and age $(P<0.001)$, DM duration $(P<0.001)$, HbAlc $(P=0.007)$, HDL-C 
TABLE 1: Clinical characteristics of the participants.

\begin{tabular}{|c|c|c|c|}
\hline Item & NDR & DR & $P$ value \\
\hline $\begin{array}{l} \\
\text { (male/female) }\end{array}$ & $1063(575 / 488)$ & $550(269 / 281)$ & \\
\hline FHD & $336(39.8 \%)$ & $200(45.9 \%)$ & 0.036 \\
\hline Age (year) & $54.09 \pm 12.71$ & $56.04 \pm 11.19$ & 0.003 \\
\hline DD (year) & $6.06 \pm 5.50$ & $10.38 \pm 6.60$ & $<0.001$ \\
\hline $\mathrm{FBG}(\mathrm{mmol} / \mathrm{L})$ & $9.15 \pm 3.62$ & $10.25 \pm 6.43$ & $<0.001$ \\
\hline $2 \mathrm{hPG}(\mathrm{mmol} / \mathrm{L})$ & $16.91 \pm 6.21$ & $18.74 \pm 8.62$ & $<0.001$ \\
\hline HbAlc (\%) & $8.26 \pm 2.24$ & $8.74 \pm 2.16$ & 0.003 \\
\hline $\mathrm{TG}(\mathrm{mmol} / \mathrm{L})$ & $2.25 \pm 2.20$ & $2.42 \pm 2.57$ & 0.060 \\
\hline $\mathrm{TC}(\mathrm{mmol} / \mathrm{L})$ & $4.89 \pm 1.24$ & $5.23 \pm 1.56$ & $<0.001$ \\
\hline $\begin{array}{l}\text { HDL-C } \\
(\mathrm{mmol} / \mathrm{L})\end{array}$ & $1.11 \pm 0.29$ & $1.16 \pm 0.33$ & 0.013 \\
\hline $\begin{array}{l}\text { LDL-C } \\
(\mathrm{mmol} / \mathrm{L})\end{array}$ & $3.11 \pm 1.00$ & $3.35 \pm 1.20$ & 0.002 \\
\hline SBP (mmHg) & $132.29 \pm 21.00$ & $139.16 \pm 23.94$ & $<0.001$ \\
\hline DBP (mmHg) & $82.75 \pm 11.32$ & $83.99 \pm 11.76$ & 0.033 \\
\hline eGFR (mL/min) & $106.25 \pm 23.32$ & $95.27 \pm 31.98$ & $<0.001$ \\
\hline $\operatorname{SCR}(\mu \mathrm{mmol} / \mathrm{L})$ & $65.16 \pm 36.72$ & $83.01 \pm 82.61$ & $<0.001$ \\
\hline BUN (mmol/L) & $6.61 \pm 7.87$ & $7.28 \pm 5.04$ & 0.062 \\
\hline
\end{tabular}

NDR: nondiabetic retinopathy; DR: diabetic retinopathy; FHD: family history of diabetes mellitus; DD: duration of diabetes mellitus; FBG: fasting blood glucose; 2hPG: 2-hour postprandial blood glucose; HbAlc: hemoglobin Alc; TG: triglyceride; TC: total cholesterol; HDL-C: high density lipoprotein-cholesterol; LDL-C: low density lipoprotein-cholesterol; SBP: systolic blood pressure; DBP: diastolic blood pressure; GFR: glomerular filtration rate; Scr: serum creatinine; BUN: blood urea nitrogen.

TABLE 2: The logistic regression analysis of the risk factors for DR.

\begin{tabular}{|c|c|c|c|}
\hline Variables & $\begin{array}{c}\text { DR } \\
\text { odds ratio }(95 \% \mathrm{CI})\end{array}$ & $P$ value & $\beta$ \\
\hline Sex & $0.933(0.545-1.599)$ & 0.802 & \\
\hline FHD & $1.186(0.693-2.029)$ & 0.534 & \\
\hline Age (year) & $0.940(0.911-0.971)$ & $<0.001^{*}$ & -0.062 \\
\hline DD (year) & $1.163(1.107-1.222)$ & $<0.001^{*}$ & 0.151 \\
\hline $\mathrm{FBG}(\mathrm{mmol} / \mathrm{L})$ & $0.979(0.885-1.082)$ & 0.676 & \\
\hline 2hPG (mmol/L) & $1.027(0.971-1.085)$ & 0.351 & \\
\hline HbAlc (\%) & $1.224(1.056-1.418)$ & $0.007^{*}$ & 0.202 \\
\hline $\mathrm{TG}(\mathrm{mmol} / \mathrm{L})$ & $1.094(0.828-1.447)$ & 0.526 & \\
\hline $\mathrm{TC}(\mathrm{mmol} / \mathrm{L})$ & $0.685(0.322-1.460)$ & 0.327 & \\
\hline HDL-C (mmol/L) & $3.884(1.191-12.672)$ & $0.024^{*}$ & 1.357 \\
\hline LDL-C (mmol/L) & $1.172(0.547-2.513)$ & 0.620 & \\
\hline SBP (mmHg) & $1.032(1.015-1.050)$ & $<0.001^{*}$ & 0.032 \\
\hline DBP (mmHg) & $0.953(0.920-0.987)$ & $0.007^{*}$ & -0.048 \\
\hline eGFR (mL/min) & $0.973(0.955-0.991)$ & $0.010^{*}$ & -0.023 \\
\hline $\mathrm{SCR}(\mu \mathrm{mmol} / \mathrm{L})$ & $1.002(0.995-1.009)$ & 0.492 & \\
\hline BUN (mmol/L) & $0.931(0.848-1.002)$ & 0.133 & \\
\hline
\end{tabular}

${ }^{*} P<0.05$.
$(P=0.024), \mathrm{SBP}(P<0.001), \mathrm{DBP}(P=0.007)$, and eGFR $(P=0.010)$. According to the severity of the disease, DR patients were divided into 3 groups: nondiabetic retinopathy (NDR), nonproliferative diabetic retinopathy (NPDR), and proliferative diabetic retinopathy (PDR) groups. The mean value of eGFR was $106.27 \pm 23.37,100.12 \pm 30.85$, and $83.33 \pm 33.77(\mathrm{~mL} / \mathrm{min})$ for the NDR, NPDR, and PDR groups, respectively.

In order to accurately study the relationship between eGFR and DR, we need to excluded the influence factors that have an effect on their relationship through stratified analysis, interaction tests, and covariate screening. First of all, we analyzed the relationship between DR and all risk factors through stratified analysis (Table 3). Each continuous variable was divided into three groups according to its value from low to high. "Sex" group was divided into "male" and "female" groups on the basis of gender. "FHD" group was divided into "No" (not having family history of DM) and "Yes" (having family history of DM) group. The results indicated that there were no confounding factors between the layers. In addition, the relationships between DR and TG and DR and DBP were not identified using univariate analysis but were observed using stratified analysis. Interaction tests were performed to detect the influence of each stratified factor on the relationship between eGFR and DR. $P \leqslant$ 0.05 means interaction exists between that factor and the relationship. The presence of effect modifiers of eGFR and DR was observed. Eligible factors, which as effect modifiers for the relationship between eGFR and DR, include SCR $(P=$ $0.003)$, SBP $(P=0.043)$, LDL-C $(P=0.025)$, TC $(P=0.005)$, and BUN $(P=0.062)(P=0.062$ means there is certain effect modification).

Covariate screening was analyzed using computer software. The screening criteria included risk factors producing $>10 \%$ change in the regression coefficient after introduction into the basic model. The results showed that the SBP, FBG, 2hPG, HbAlc, and the DM duration met the filter criteria (OR change was $13.5,13.4,16.5,18.5$, and 39.0 , resp.).

After the adjustment of the variables affecting the relationship between eGFR and DR, result of univariate analysis suggested that eGFR remained significantly associated with DR $(P<0.001)$ (Table 4$)$. In addition, smooth curve fitting was performed after the adjustment of all variables, and the resultant curve exhibited a two-stage change and a breakpoint (Figure 1). When the eGFR value was more than the point, the risk of DR was low; however if the value was less than the point, the risk of DR significantly increased. The saturation effects were analyzed based on the curve, and the data indicated that the inflection point was $99.4 \mathrm{~mL} / \mathrm{min}$ (34th percentile). Before and after the adjustment of the covariates, the logarithmic likelihood ratio test $P$ value decreased from 0.050 to 0.036 (Table 5). The results of the ROC curve are shown in Figure 2. The area under the ROC curve (AUC) was 0.591815 . Combined with clinical significance, the cut-off value remained $99.4 \%$. The sensitivity and specificity of this point were $42.77 \%$ and $70.25 \%$, respectively. 
TABLE 3: Stratified analysis of all variables and interaction tests.

\begin{tabular}{|c|c|c|c|}
\hline & $N(\%)$ & $\mathrm{DR}$ & $P$ value of interaction \\
\hline Sex & & & 0.453 \\
\hline Male & $518(53.7 \%)$ & $0.984(0.977,0.991)<0.001$ & \\
\hline Female & $446(46.3 \%)$ & $0.988(0.981,0.995) 0.001$ & \\
\hline FHD & & & 0.839 \\
\hline No & $427(57.1 \%)$ & $0.984(0.977,0.991)<0.001$ & \\
\hline Yes & $321(42.9 \%)$ & $0.983(0.974,0.992)<0.001$ & \\
\hline BUN & & & 0.062 \\
\hline Low & $317(33.0 \%)$ & $0.986(0.972,0.999) 0.039$ & \\
\hline Medium & $324(33.7 \%)$ & $1.003(0.989,1.018) 0.654$ & \\
\hline High & $321(33.4 \%)$ & $0.984(0.977,0.991)<0.001$ & \\
\hline SCR & & & $0.030^{*}$ \\
\hline Low & $300(31.1 \%)$ & $1.002(0.987,1.018) 0.802$ & \\
\hline Medium & $338(35.1 \%)$ & $0.979(0.964,0.995) 0.008$ & \\
\hline High & $326(33.8 \%)$ & $0.979(0.971,0.986)<0.001$ & \\
\hline DBP & & & 0.411 \\
\hline Low & $169(25.9 \%)$ & $0.980(0.968,0.992)<0.001$ & \\
\hline Medium & $242(37.1 \%)$ & $0.990(0.980,0.999) 0.036$ & \\
\hline High & $242(37.1 \%)$ & $0.983(0.974,0.993)<0.001$ & \\
\hline SBP & & & $0.043^{*}$ \\
\hline Low & $138(21.1 \%)$ & $0.983(0.968,0.998) 0.024$ & \\
\hline Medium & $232(35.5 \%)$ & $0.999(0.987,1.010) 0.813$ & \\
\hline High & $284(43.4 \%)$ & $0.981(0.973,0.990)<0.001$ & \\
\hline LDL & & & $0.025^{*}$ \\
\hline Low & $296(34.1 \%)$ & $0.992(0.983,1.001) 0.068$ & \\
\hline Medium & $284(32.7 \%)$ & $0.988(0.978,0.999) 0.029$ & \\
\hline High & $289(33.3 \%)$ & $0.975(0.966,0.985)<0.001$ & \\
\hline HDL & & & 0.618 \\
\hline Low & $291(33.5 \%)$ & $0.986(0.977,0.994) 0.001$ & \\
\hline Medium & $289(33.3 \%)$ & $0.981(0.971,0.991)<0.001$ & \\
\hline High & $288(33.2 \%)$ & $0.987(0.978,0.996) 0.006$ & \\
\hline $\mathrm{TC}$ & & & $0.005^{*}$ \\
\hline Low & $297(34.2 \%)$ & $0.996(0.987,1.005) 0.377$ & \\
\hline Medium & $289(33.3 \%)$ & $0.982(0.972,0.993)<0.001$ & \\
\hline High & $283(32.6 \%)$ & $0.976(0.966,0.985)<0.001$ & \\
\hline TG & & & 0.526 \\
\hline Low & $294(33.8 \%)$ & $0.989(0.980,0.998) 0.018$ & \\
\hline Medium & $290(33.3 \%)$ & $0.985(0.976,0.994) 0.001$ & \\
\hline High & $286(32.9 \%)$ & $0.981(0.972,0.991)<0.001$ & \\
\hline HbAlc & & & 0.865 \\
\hline Low & $237(31.7 \%)$ & $0.980(0.970,0.990)<0.001$ & \\
\hline Medium & $252(33.7 \%)$ & $0.984(0.972,0.995) 0.004$ & \\
\hline High & $259(34.6 \%)$ & $0.983(0.971,0.9958) 0.005$ & \\
\hline PBG & & & 0.247 \\
\hline Low & $211(28.7 \%)$ & $0.976(0.965,0.986)<0.001$ & \\
\hline Medium & $252(34.2 \%)$ & $0.983(0.973,0.993) 0.001$ & \\
\hline High & $273(37.1 \%)$ & $0.988(0.978,0.999) 0.027$ & \\
\hline FBG & & & 0.206 \\
\hline Low & $284(31.3 \%)$ & $0.978(0.970,0.987)<0.001$ & \\
\hline Medium & $297(32.8 \%)$ & $0.991(0.9808,1.002) 0.093$ & \\
\hline High & $325(35.9 \%)$ & $0.981(0.971,0.991)<0.001$ & \\
\hline
\end{tabular}


TABle 3: Continued.

\begin{tabular}{lccc}
\hline \multicolumn{1}{c}{$N(\%)$} & DR & $P$ value of interaction \\
\hline DD & & & 0.213 \\
Low & $255(31.9 \%)$ & $0.995(0.982,1.008) 0.443$ & $0.995(0.984,1.006) 0.360$ \\
$\quad$ Medium & $246(30.8 \%)$ & $0.984(0.976,0.993)<0.001$ & \\
$\quad$ High & $298(37.3 \%)$ & & $0.985(0.975,0.996) 0.005$ \\
AGE & & $0.985(0.976,0.995) 0.003$ & \\
Low & $317(32.9 \%)$ & $0.981(0.972,0.990)<0.001$ & \\
Medium & $318(33.0 \%)$ &
\end{tabular}

${ }^{*} P<0.05$.

Each continuous variable was divided into three groups according to its value from low to high; "Sex" group was divided into male and female; "FHD" group was divided into No (not having family history of DM) and Yes (having family history of DM).

TABLE 4: Correlation analysis after adjustment of the effect modifier.

\begin{tabular}{lcc}
\hline & Statistics & DR \\
\hline GFR & $102.45 \pm 27.09$ & $0.971(0.954,0.988)<0.001$ \\
\hline
\end{tabular}

Adjustment variables: duration of DM, FBG, 2hPG, HbAlc, LDL, TC, BUN, SCR, and SBP.

\section{Discussion}

Diabetic retinopathy is one of the most common microvascular complications of DM [15]. With an increase in morbidity of DM, the prevalence of DR has increased yearly, becoming the leading cause of visual impairment and blindness in working-aged people. Thus, it is important to characterize the incidence of DR and identify key factors for predicting it.

Similar to other hospital-based epidemiologic studies in urban China [16], the prevalence of DR in the this study was $34.1 \%$, which is much higher than in Europe, the United States, South Korea, and the other developed countries [17, 18]. This potentially reflects the higher incidence of DM in China [2] and the poor knowledge or less attention to the complications of DM, particularly DR [19]. To reduce the prevalence of DM and slow down the progression of DR, further efforts should be exerted on concerning the public health education of DM and the complications of it.

Many studies have described the risk factors for DR, which primarily include the extended duration of DM, old age, hyperglycemia, hyperlipidemia, proteinuria, severe obesity, alcohol consumption, genetic factors, and the expression of a variety of hormones, such as growth hormone [20-23]. Similarly, the present study confirmed that age, extended duration of DM, high blood sugar, high blood pressure, and hyperlipidemia were significantly associated with DR. In addition, we also showed that eGFR was negatively correlated with DR, consistent with previous studies [13, 24]. These results suggest that measurements of eGFR might help to predict the early stage of DR.

Then we successively conducted stratified analysis, interaction test, and covariate screening to get rid of the factors that affect the relationship between DR and eGFR. After the adjustment of these influence factors, the results still suggested that eGFR was significantly associated with DR. The graphical representation of the relationship between them

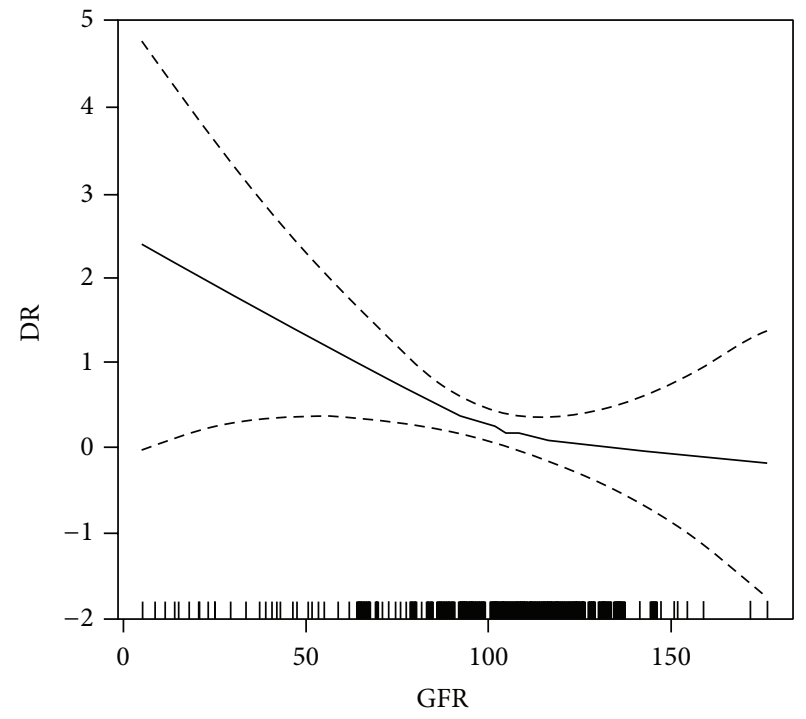

FIgURE 1: Smooth curve fitting of DR and eGFR. Adjustment variables: duration of DM, FBG, $2 \mathrm{hPG}, \mathrm{HbAlc}, \mathrm{LDL}, \mathrm{TC}, \mathrm{BUN}, \mathrm{SCR}$, and SBP.

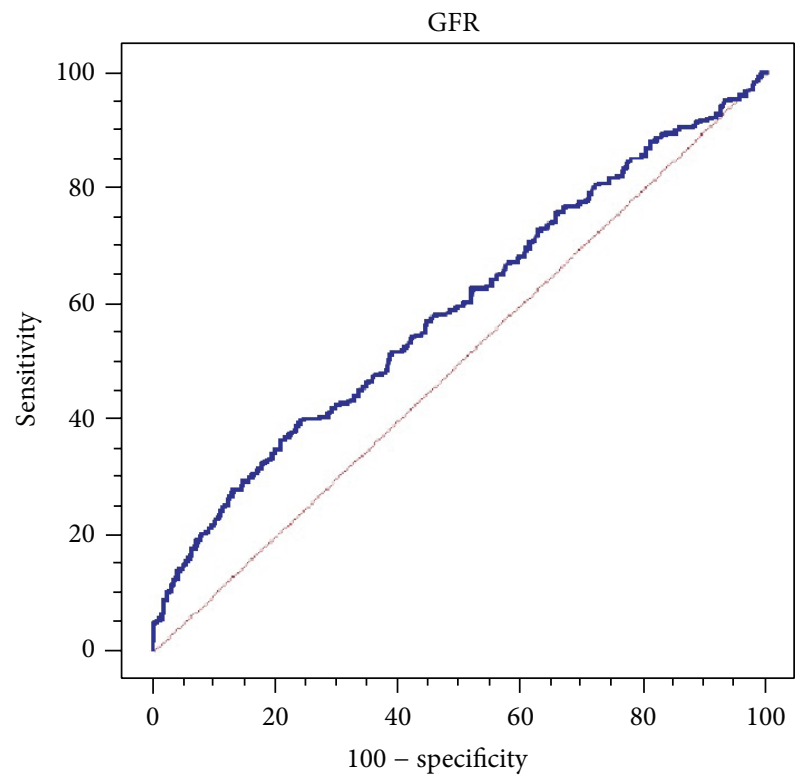

FIGURE 2: ROC curve of the relationship between DR and eGFR. 
TABLE 5: Saturation effect analysis before and after adjustment of the effect modifier.

\begin{tabular}{llcr}
\hline & & Before adjustment & After adjustment \\
\hline Model I & OR value & $0.985(0.980,0.990)<0.001$ & $0.988(0.974,1.002) 0.089$ \\
\hline \multirow{3}{*}{ Model II } & Breakpoint $(\mathrm{K})$ & $97.330(31$ st percentile $)$ & $99.400(34$ th percentile $)$ \\
& OR1 $(<99.400)$ & $0.979(0.971,0.987)<0.001$ & $0.965(0.939,0.991) 0.008$ \\
& OR2 $(>99.400)$ & $0.995(0.984,1.006) 0.351$ & $1.003(0.983,1.023) 0.779$ \\
& OR2/OR1 & $1.016(1.00,1.032) 0.050$ & $1.040(1.002,1.078) 0.037$ \\
& Logarithmic likelihood ratio test $P$ value & 0.050 & 0.036 \\
\hline
\end{tabular}

Adjustment variables: duration of DM, FBG, 2hPG, HbAlc, LDL, TC, BUN, SCR, and SBP.

displayed a two-stage pattern and that the value of eGFR less than or equal to $99.4 \mathrm{~mL} / \mathrm{min}$ was significantly correlated with DR. The AUC was 0.591815, a value between 0.5 and 0.7 , indicating that the diagnostic value was not very useful, likely reflecting a bias in this study based on the fact that absence of the related variables with GFR, like urine protein or microalbumin. In addition, recent studies have shown that the incidence of kidney disease in some diabetic patients was independent of DN, referred to as nondiabetic renal disease (NDRD) $[25,26]$. The renal tissue pathological examination revealed that both DN and NDRD reduced GFR levels. DN and DR normally appear in the same person at the same time, so we cannot artificially exclude the patients suffering from DN. However, NDRD patients may consist in the study population and their results could reduce the value of GFR, which affected the results of experimental. Therefore, there may have been selection bias in the study objects.

GFR is an important indicator of kidney function and an important factor in the diagnosis of diabetic nephropathy [27]. Although the direct relationship between GFR and other microangiopathy of DM has not been fully established, high levels of GFR have been negatively associated with the onset of macroangiopathy, such as coronary artery disease [28, 29]. A recent study has also confirmed that DR is closely associated with regional arterial stiffness [30]. Many studies have confirmed that both DR and DN are microvascular complications with similar pathological bases, associated with DM [31-33]. A report from the Atherosclerosis Risk in Communities (ARIC) Study demonstrated that retinopathy and renal dysfunction have a strong association, independent of age, diabetes, hypertension, and other risk factors. Systemic markers of inflammation and endothelial dysfunction associated with retinal vascular abnormalities could contribute to the development of kidney disease [34]. Animal studies have also shown that pathological changes in the retina are highly associated with renal microcirculation [35].

For DM patients, DR and DN have a common pathological basis and a similar course of evolution. Hyperglycemia causes glomerular hyperperfusion and high filtration, leading to an increase of GFR during the early stages of T2DM [36]. The accumulation of advanced glycation end products, due to hyperglycemia, promotes mesangial proliferation and basement membrane thickening in the glomerulus. In addition, the activation of the polyol pathway, the protein kinase $\mathrm{C}$ pathway, the pentose phosphate pathway [37], oxidative stress, and various cytokines cause a range of variations in kidney, which include capillary obstruction, a reduction of podocyte proliferation, the loss of the urinary proteins, and a decline in renal function. With further thickening of the glomerular basement membrane, the mesangial matrix increases, resulting in the appearance of cracks and an increase in urinary protein leakage [38]. Accordingly, changes in GFR appear earlier than those in urine protein levels, and these changes remain throughout the entire course of DM.

The pathological mechanisms described above are similar to those observed in the retina. As in the kidney, the high blood sugar exerts deleterious effects on the retina, which include the apoptosis of Muller cells, ganglion cells [39], and pericytes, the thickening of the capillary basement membrane, and the proliferation of endothelial cells in the retina. These effects lead to pathological changes in DR, including nonperfusive capillaries, the appearance of microaneurysms, and exudation. Hence, GFR not only may be an important clinical marker for DN, but also could be correlated with DR.

Taken together, these data highlight the use of eGFR as a predictor of DR. However, the results of the present study are important to the primary hospital for DR screening, especially to the ones with limited resources in China. But there are still some limitations. First, this study was a retrospective case-control study, including hospital-based patients with DM. Second, DR was diagnosed using ophthalmoscopy and fundus photography, but not fundus fluorescein angiography (FFA). Third, the GFR values were estimated by formula, and some of the factors associated with GFR, such as urinary protein excretion or microalbuminuria, were not included in this study. Therefore, a long-term follow-up study should be performed in the future. Moreover, more factors should be included in this study to provide an in-depth examination of the relationship and mechanism of GFR and DR.

In summary, the results of the present study indicate that GFR may have certain implications for DR, which is important to DR screening in primary hospital of China, especially in poverty-stricken areas. Additional studies are needed to explore the mechanisms that couple GFR and DR.

\section{Conflict of Interests}

There is no conflict of interests in this research.

\section{Authors' Contribution}

Jingyang $\mathrm{Wu}$ and Lei Liu were responsible for analysis and interpretation of data and drafted a part of the paper. Weiping 
Teng and Lei Chen have contributed to the design of the study. Jin Geng and Limin Liu took part in analyzing data and drafting a part of the paper. All authors reviewed the paper.

\section{Acknowledgments}

This study was supported by National Natural Science Foundation of China (81300783) and Important Platform of Science and Technology for the University in Liaoning Province (16010).

\section{References}

[1] H. King, R. E. Aubert, and W. H. Herman, "Global burden of diabetes, 1995-2025: prevalence, numerical estimates, and projections," Diabetes Care, vol. 21, no. 9, pp. 1414-1431, 1998.

[2] K.-H. Yoon, J.-H. Lee, J.-W. Kim et al., "Epidemic obesity and type 2 diabetes in Asia," The Lancet, vol. 368, no. 9548, pp. 1681$1688,2006$.

[3] L. Liu, X. Wu, J. Geng, Z. Yuan, Z. Shan, and L. Chen, "Prevalence of diabetic retinopathy in mainland china: a metaanalysis," PLoS ONE, vol. 7, no. 9, Article ID e45264, 2012.

[4] M. Malek, M. E. Khamseh, R. Aghili, Z. Emami, L. Najafi, and H. R. Baradaran, "Medical management of diabetic retinopathy: an overview," Archives of Iranian Medicine, vol. 15, no. 10, pp. 635640, 2012.

[5] P. P. C. Chiang, E. L. Lamoureux, C. Y. Cheung et al., "Racial differences in the prevalence of diabetes but not diabetic retinopathy in a multi-ethnic asian population," Investigative Ophthalmology and Visual Science, vol. 52, no. 10, pp. 7586-7592, 2011.

[6] E. L. Lamoureux, E. S. Tai, J. Thumboo et al., "Impact of diabetic retinopathy on vision-specific function," Ophthalmology, vol. 117 , no. 4, pp. 757-765, 2010.

[7] Y. Zheng, M. He, and N. Congdon, "The worldwide epidemic of diabetic retinopathy," Indian Journal of Ophthalmology, vol. 60, no. 5, pp. 428-431, 2012.

[8] S. C. Satchell and J. E. Tooke, "What is the mechanism of microalbuminuria in diabetes: a role for the glomerular endothelium?” Diabetologia, vol. 51, no. 5, pp. 714-725, 2008.

[9] K. Krzyzanowska, F. Mittermayer, G. H. Schernthaner et al., "Renal function but not asymmetric dimethylarginine is independently associated with retinopathy in type 2 diabetes," Cardiology Research and Practice, vol. 2011, Article ID 260191, 6 pages, 2011.

[10] B.-B. He, L. Wei, Y.-J. Gu et al., "Factors associated with diabetic retinopathy in chinese patients with type 2 diabetes mellitus," International Journal of Endocrinology, vol. 2012, Article ID 157940, 8 pages, 2012.

[11] WHO, Definition and Diagnosis of Diabetes Mellitus and Intermediate Hyperglycemia: Report of a WHO/IDF Consultation, World Health Organization, Geneva, Switzerland, 2006.

[12] C. P. Wilkinson, F. L. Ferris III, R. E. Klein et al., "Proposed international clinical diabetic retinopathy and diabetic macular edema disease severity scales," Ophthalmology, vol. 110, no. 9, pp. 1677-1682, 2003.

[13] H.-H. Parving, C. E. Mogensen, M. C. Thomas, B. M. Brenner, and M. E. Cooper, "Poor prognosis in proteinuric type 2 diabetic patients with retinopathy: insights from the RENAAL study," The Quarterly Journal of Medicine, vol. 98, no. 2, pp. 119-126, 2005.
[14] M. Vučić Lovrenčic, V. Radišić Biljak, S. Božičević, M. Prašek, P. Pavković, and M. Knotek, "Estimating glomerular filtration rate (GFR) in diabetes: the performance of MDRD and CKDEPI equations in patients with various degrees of albuminuria," Clinical Biochemistry, vol. 45, no. 18, pp. 1694-1696, 2012.

[15] E. M. Kohner, S. J. Aldington, I. M. Stratton et al., "United kingdom prospective diabetes study, 30: diabetic retinopathy at diagnosis of non-insulin-dependent diabetes mellitus and associated risk factors," Archives of Ophthalmology, vol. 116, no. 3, pp. 297-303, 1998.

[16] B. Zhang, H.-D. Xiang, W.-B. Mao et al., "Epidemiological survey of chronic vascular complications of type 2 diabetic in-patients in four municipalities," Acta Academiae Medicinae Sinicae, vol. 24, no. 5, pp. 452-456, 2002.

[17] K. Kostev and W. Rathmann, "Diabetic retinopathy at diagnosis of type 2 diabetes in the UK: a database analysis," Diabetologia, vol. 56, no. 1, pp. 109-111, 2013.

[18] J.-H. Kim, H.-S. Kwon, Y.-M. Park et al., "Prevalence and associated factors of diabetic retinopathy in Rural Korea: the chungju metabolic disease cohort study," Journal of Korean Medical Science, vol. 26, no. 8, pp. 1068-1073, 2011.

[19] R. A. Steinman and B. K. Birshtein, "Treatment and awareness of type 2 diabetes in Beijing, China, compared to New York," The Diabetes Educator, vol. 33, no. 2, pp. 282-290, 2007.

[20] W. T. Seo, S. O. Song, S. Y. Kim, Y. S. Choi, H. R. Jang, and S. J. Lee, "The risk factors of diabetic retinopathy in NIDDM patients," Journal of the Korean Diabetes Association, vol. 23, no. 2, pp. 162-171, 1999.

[21] H. K. Kim, T. S. Oh, S. M. Lee, and J. B. Lee, "The initial fundus examination and severity of diabetic retinopathy at a primary eye clinic," Journal of the Korean Ophthalmological Society, vol. 46, pp. 982-988, 2005.

[22] J. H. Jin, S. J. Lee, H. S. Lee, and S. D. Kim, "Prognostic factors of visual acuity in diabetes mellitus," Journal of the Korean Ophthalmological Society, vol. 47, pp. 755-762, 2006.

[23] C. Y. Choi, J. Y. Lee, J. M. Kim, K. H. Park, and E. J. Rhee, "Quantitative analysis of aqueous flare in diabetic patients," Journal of the Korean Ophthalmological Society, vol. 49, pp. 1954-1960, 2008.

[24] H. J. Kramer, Q. D. Nguyen, G. Curhan, and C.-Y. Hsu, "Renal insufficiency in the absence of albuminuria and retinopathy among adults with type 2 diabetes mellitus," The Journal of the American Medical Association, vol. 289, no. 24, pp. 3273-3277, 2003.

[25] E. Amoah, J. L. Glickman, C. D. Malchoff, B. C. Sturgill, D. L. Kaiser, and W. K. Bolton, "Clinical identification of nondiabetic renal disease in diabetic patients with type I and type II disease presenting with renal dysfunction," The American Journal of Nephrology, vol. 8, no. 3, pp. 204-211, 1988.

[26] L. Zhuo, G. Zou, W. Li, J. Lu, and W. Ren, "Prevalence of diabetic nephropathy complicating non-diabetic renal disease among Chinese patients with type 2 diabetes mellitus," European Journal of Medical Research, vol. 18, no. 1, article 4, 2013.

[27] J. S. Alwakeel, A. C. Isnani, A. Alsuwaida et al., "Factors affecting the progression of diabetic nephropathy and its complications: a single-center experience in Saudi Arabia," Annals of Saudi Medicine, vol. 31, no. 3, pp. 236-242, 2011.

[28] A. K. Bello, B. Hemmelgarn, A. Lloyd et al., "Associations among estimated glomerular filtration rate, proteinuria, and adverse cardiovascular outcomes," Clinical Journal of the American Society of Nephrology, vol. 6, no. 6, pp. 1418-1426, 2011. 
[29] H. Ito, Y. Komatsu, M. Mifune et al., “The estimated GFR, but not the stage of diabetic nephropathy graded by the urinary albumin excretion, is associated with the carotid intima-media thickness in patients with type 2 diabetes mellitus: a crosssectional study," Cardiovascular Diabetology, vol. 9, article 18, 2010.

[30] W. J. Kim, C.-Y. Park, S. E. Park et al., "The association between regional arterial stiffness and diabetic retinopathy in type 2 diabetes," Atherosclerosis, vol. 225, no. 1, pp. 237-241, 2012.

[31] M. R. Manaviat, M. Afkhami, and M. R. Shoja, "Retinopathy and microalbuminuria in type II diabetic patients," $B M C$ Ophthalmology, vol. 4, article 9, 2004.

[32] A. Girach and L. Vignati, "Diabetic microvascular complications-can the presence of one predict the development of another?" Journal of Diabetes and its Complications, vol. 20, no. 4, pp. 228-237, 2006.

[33] M. C. Boelter, J. L. Gross, L. H. Canani et al., "Proliferative diabetic retinopathy is associated with microalbuminuria in patients with type 2 diabetes," Brazilian Journal of Medical and Biological Research, vol. 39, no. 8, pp. 1033-1039, 2006.

[34] C. Sabanayagam, A. Shankar, D. Koh et al., "Retinal microvascular caliber and chronic kidney disease in an Asian population," The American Journal of Epidemiology, vol. 169, no. 5, pp. 625$632,2009$.

[35] T. Y. Wong, R. Klein, F. J. Nieto et al., "Retinal microvascular abnormalities and 10-year cardiovascular mortality: a population-based case-control study," Ophthalmology, vol. 110, no. 5, pp. 933-940, 2003.

[36] S. P. Silveiro, R. Friedman, M. J. De Azevedo, L. H. Canani, and J. L. Gross, "Five-year prospective study of glomerular filtration rate and albumin excretion rate in normofiltering and hyperfiltering normoalbuminuric NIDDM patients," Diabetes Care, vol. 19, no. 2, pp. 171-174, 1996.

[37] H. J. Goldberg, J. Scholey, and I. G. Fantus, "Glucosamine activates the plasminogen activator inhibitor 1 gene promoter through Sp1 DNA binding sites in glomerular mesangial cells," Diabetes, vol. 49, no. 5, pp. 863-871, 2000.

[38] A. E. Raptis and G. Viberti, "Pathogenesis of diabetic nephropathy," Experimental and Clinical Endocrinology \& Diabetes, vol. 109, supplement 2, pp. S424-S437, 2001.

[39] H. P. Hammes, H. J. Federoff, and M. Brownlee, "Nerve growth factor prevents both neuroretinal programmed cell death and capillary pathology in experimental diabetes," Molecular Medicine, vol. 1, no. 5, pp. 527-534, 1995. 


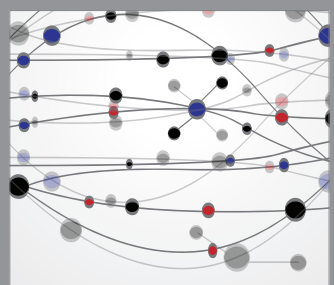

The Scientific World Journal
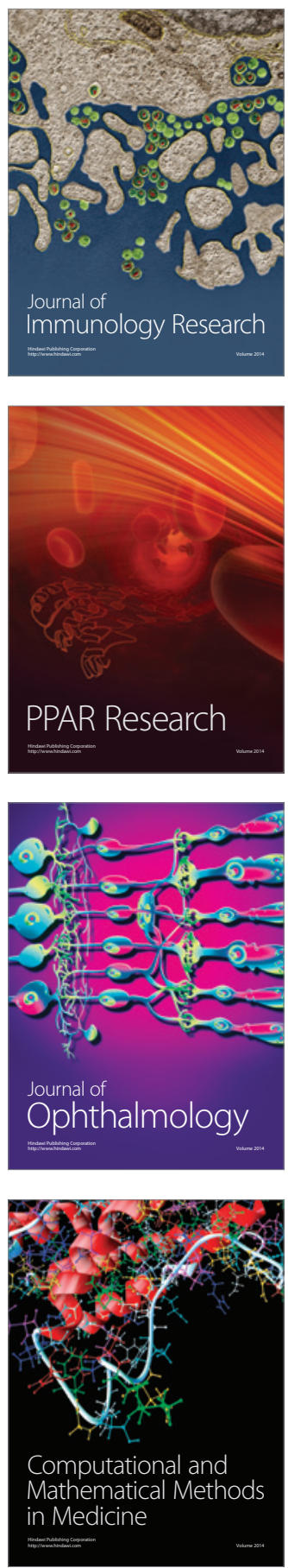

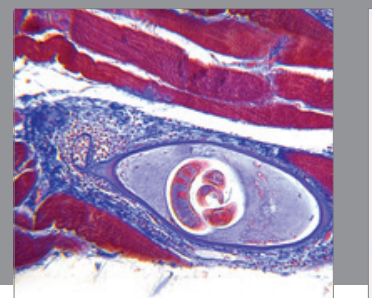

Gastroenterology

Research and Practice
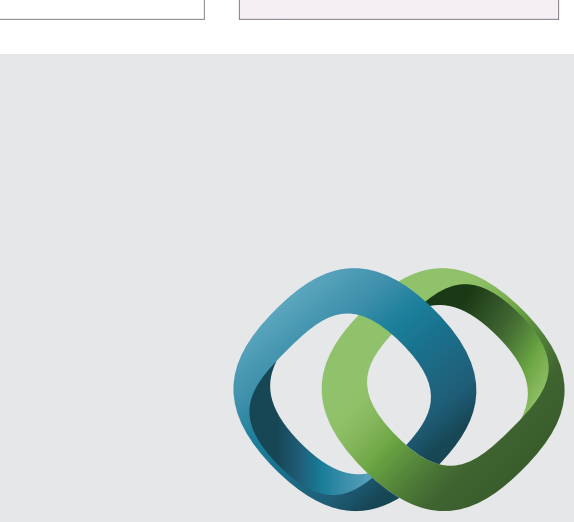

\section{Hindawi}

Submit your manuscripts at

http://www.hindawi.com
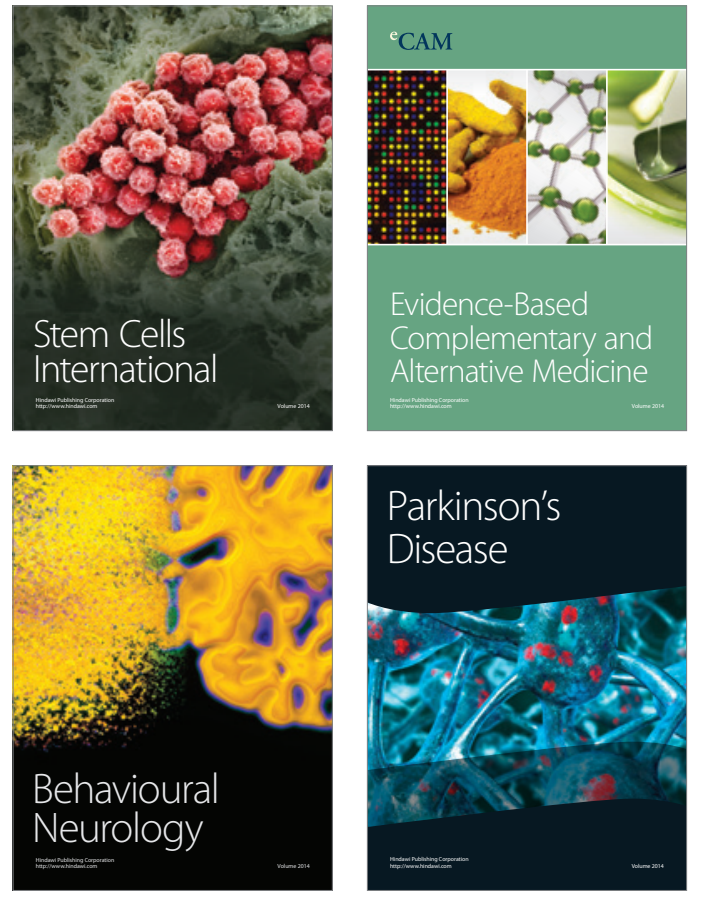
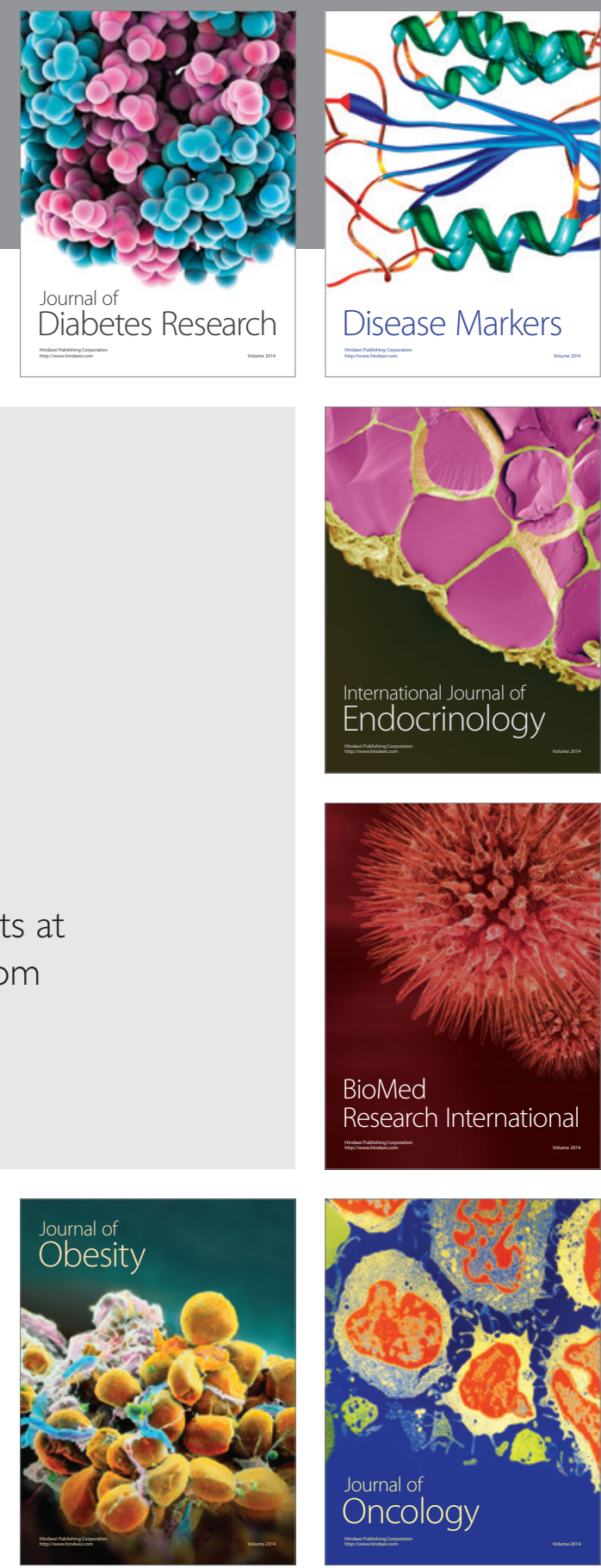

Disease Markers
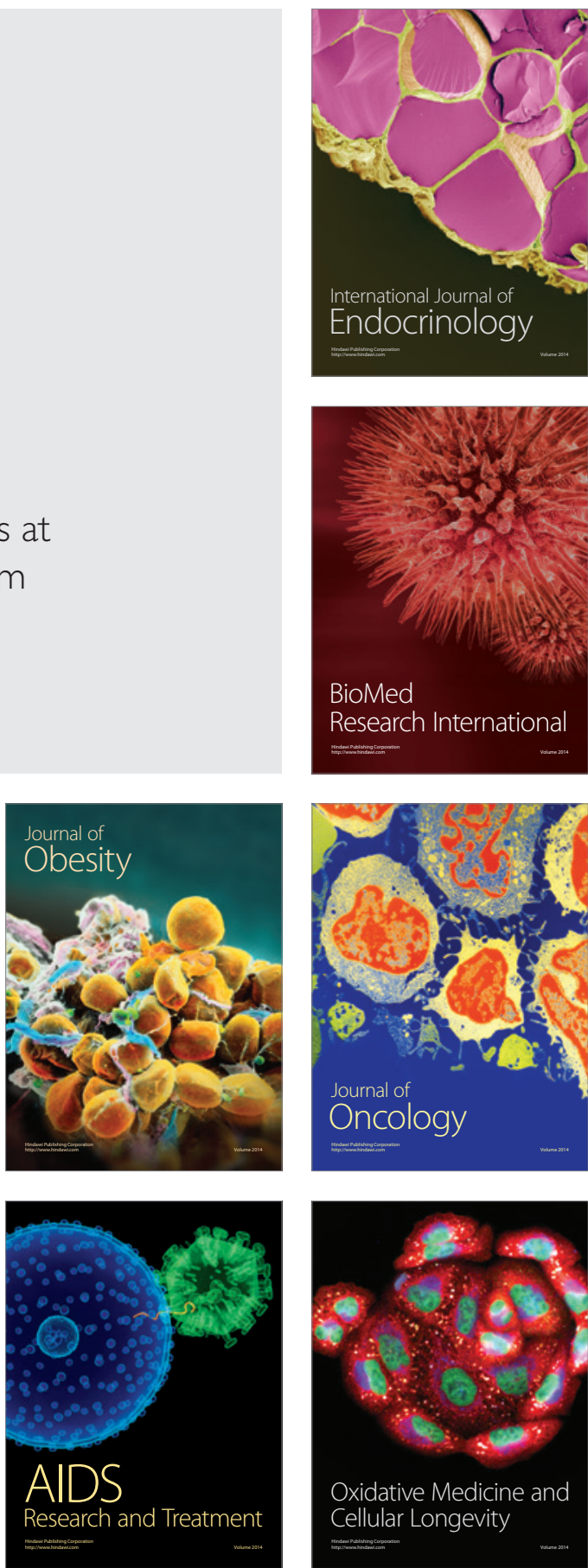